\title{
GBESS: An Energy-Saving Cluster Scheduling based on Grouping and Balancing
}

\author{
Shang Qiuli ${ }^{1}$, Liu Bingshuang ${ }^{1}$, Tu Bo ${ }^{1+}$, Dai Shuaifu ${ }^{1}$ and Zhang Jianyu ${ }^{1}$ \\ ${ }^{1}$ Seven North Road No.42 Building 16, Changping District, Beijing, China
}

\begin{abstract}
With the rapid development of Big Data and IoT, the scale of server cluster in enterprise data center is increasing sharply. In order to deal with the issue of energy consumption, this paper proposes a Grouping and Balancing based Energydfiantiog \$ \$ reduces energy consumption by grouping the nodes of cluster into hot group and cold group; 2) It creates slack space for energy-saving grouping and ensures the lower bound of QoS by load balancing the hot group. Experimental evaluation shows that GBESS can reduce the energy consumption by $12.1 \%$ on average without sacrificing QoS. Furthermore, the energy efficiency of GBESS can improve along with the cluster scale growing.
\end{abstract}

Keywords: Energy-saving, Scheduling, Load balance

\section{Introduction}

With the rapid development of Big Data and IoT (Internet of Things), the size of computing or storage cluster in enterprise data center is increasing sharply. The energy consumption of server cluster is causing great growth of electricity cost and loss of system robustness. Therefore, the issue of cluster energy consumption has aroused wide concern in both academia and industry [1][2][3][4].

Nowadays, the energy-saving scheduling algorithms on system-level of servers are mostly based on the proven techniques including DPM (Dynamic Power Management) [5][6] and DVS (Dynamic Voltage Scaling) [7]. On aspect of working mechanisms, both DPM and DVS are mainly used in controlling the energy consumption of single server or device, which might not be applicable for the scheduling of server cluster. The energy-saving cluster scheduling is a hot topic in both academia and industry. The popular strategies include PID (Proportional Integral Derivative feedback control) [8] and LC (Load Concentration strategy) [9], etc. However, as the cluster structure going complicated and the service going diversified, the limitations of these strategies are becoming more and more obvious.

In order to deal with these problems, this paper presents a Grouping and Balancing based Energy-Saving Scheduling (GBESS). GBESS is a double level strategy: 1) It reduces energy consumption by grouping the nodes of cluster into hot group and cold group; 2) It creates slack space for energy-saving grouping and ensures the lower bound of QoS (Quality of Service) by load balancing the hot group.

\section{Method}

\subsection{Idea}

The proposed method consists of two levels:

Level I: Grouping. According to global load of the cluster, it divides the nodes into hot group and cold group with dynamic adjustment.

+ Corresponding author. Tel.: +86-010-80759188; fax: +86-010-62190366.

E-mail address: tubo@ chanct.com. 
Level II: Load Balancing. According to the running data of each node, namely the resource utilization, it schedules the tasks to hot group by load balance strategy.

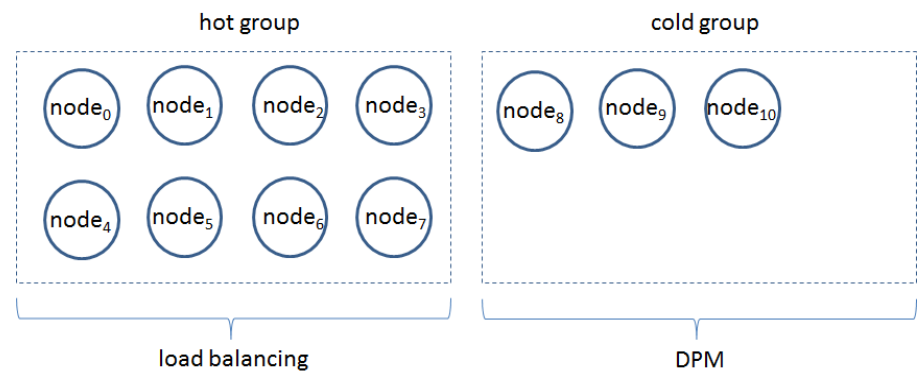

Fig. 1: Double level strategy of GBESS.

\subsection{Level I: Grouping}

According to the global load, GBESS divides the nodes into hot group and cold group. The nodes in hot group runs on line and processes new coming tasks. The hot group is scheduled by load balance strategy which is described in Level II. It further turns the nodes of cold group into sleep by DPM.

The method can adjust the grouping adaptively with the workload fluctuating. If the global load increases and exceeds the overload threshold, it selects the node with the highest load in cold group ( node $_{i+1}$ ) and then adds node $_{i+1}$ into hot group. If the global load decreases and goes lower than the idling threshold, it selects the node with the lowest load in hot group ( node $_{i}$ ) and then adds node $_{i}$ into cold group. The adjustment process is shown in Fig. 2.

\subsection{Level II: Load Balancing}

Some studies have proved that the variation between the actual execution time and the worst-case execution time of real-time tasks is $87 \%$ approximately [10]. Therefore, there will be considerable dynamic slack time when the tasks being executed. Essentially, the scheduling method based on grouping in Level I tries to exploit and squeeze the slack space to save energy. The load balancing level can further squeeze the slack space for the grouping level in order to achieve the unification of global strategy and partial strategy.

According to the running data of each node, namely the resource utilization, it schedules the tasks to hot group by load balance strategy. When new task coming, the Level II scheduling calculates the weights of nodes in hot group by load balancing and then schedules the node with the lowest weight to process the new coming task.

For example, a specific scheduling algorithm is shown as follow [11]:

1. Assume that the number of nodes in hot group at the moment is $N$, the CPU utilization and memory utilization of node $s_{i}$ is $c p u_{i}$ and buffer $r_{i}$, then the load of $s_{i}$ is $l_{i}$.

$$
l_{i}=\alpha^{*} c p u_{i}+(1-\alpha) * \text { buffer }_{i}
$$

2. It randomly selects $k$ nodes from hot group as node set $\bar{S}$, then the load of the selected nodes is $l_{1}, l_{2}, \ldots, l_{k}$.

$$
\begin{aligned}
& l_{\text {total }}=\sum_{i=1}^{k} l_{i} \\
& x_{i}=\frac{l_{\text {total }}-l_{i}}{l_{\text {total }}}
\end{aligned}
$$

The weight of node $s_{i}$ in $\bar{S}$ is $p_{i}$.

$$
p_{i}=\frac{x_{i}}{\sum_{i=1}^{k} x_{i}}, \quad s_{i} \in \bar{S}
$$

3. It selects the node with lowest weight $s_{x}$ from $\bar{S}$ to process the new coming task.

$$
w_{i}=\left\{\begin{array}{l}
1, i=x \\
0, \text { otherwise }
\end{array}\right.
$$




\subsection{Implementation}

The implementation of GBESS includes the following parts. (1) The system initialization function is Initialization () which initializes the node grouping, overload and idling thresholds, running data collection time, and further sets up the module Monitoring(). (2) The function Monitoring() collects the running data of each node periodically and evaluates the global load value. (3) The function Grouping() adjusts the grouping dynamically according to global load and the thresholds. (4) The function Load_balancing() schedules the nodes in hot group by load balancing based on resource utilization (running data) of each node, and further generates scheduling weights. (5) Timers: Sleeping_Timer() calculates the break-even time to switch the spare nodes into sleep mode; Collecting_Timer() sets the collecting period of Monitoring().

The procedure of GBESS is presented in Fig. 2.

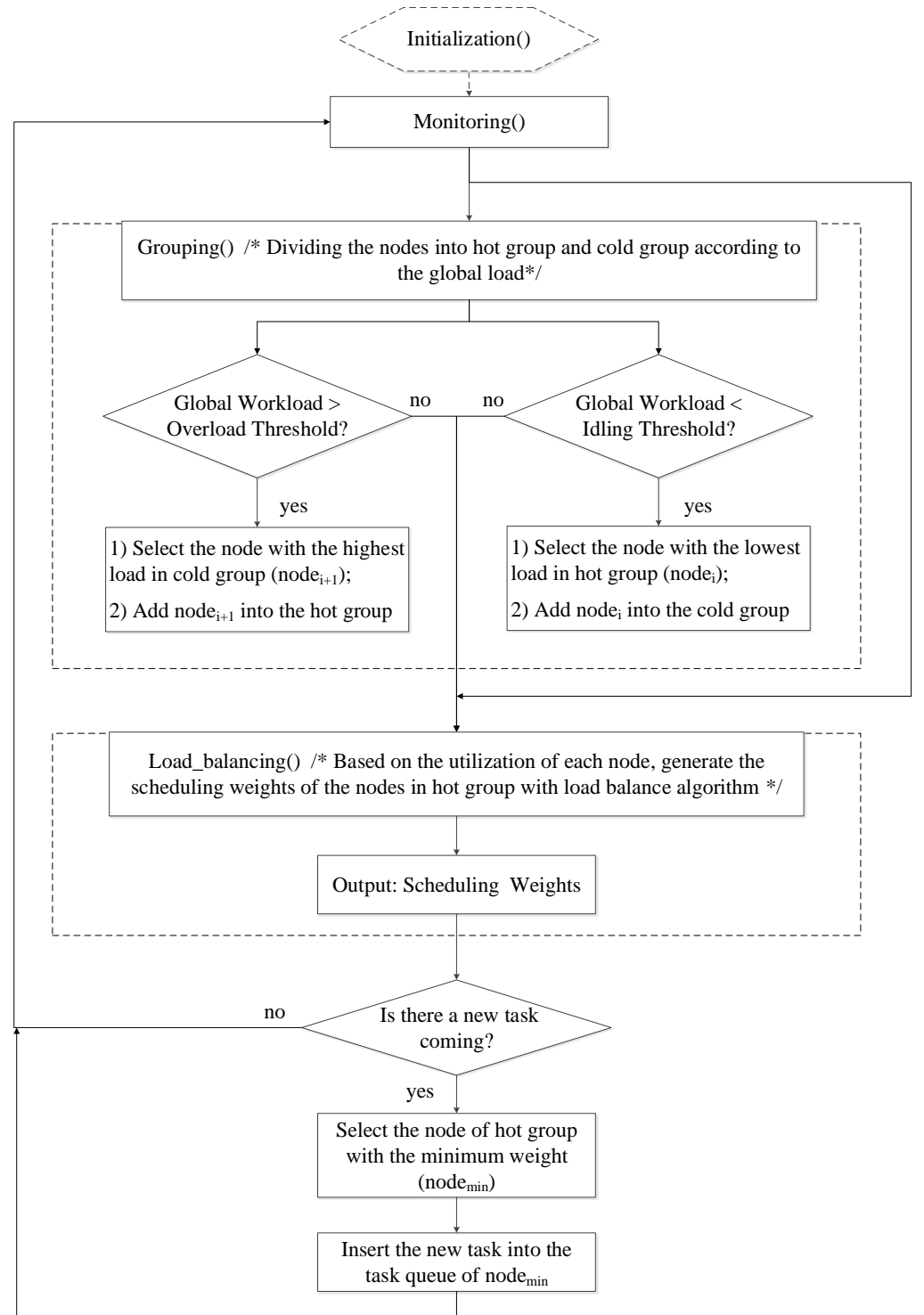

Fig. 2: The procedure of GBESS.

\section{Evaluation}

\subsection{Experimental Setup}

Fig. 3 shows the experimental testbed built to evaluate the performance of GBESS. The testbed simulates a real-time network traffic processing cluster. The network topology uses a SDN-like architecture. In implementation, GBESS runs on an independent controller which interconnects with the load distributing device and the cluster nodes via management network. The load distributing device in traffic network acts as a flow scheduler of SDN. A monitor agent is deployed on each node to response the collection requests of controller. 
The input of GBESS is the running data of nodes collected by the monitoring module periodically, including CPU utilization, memory utilization, and packet drop rate, etc. In this experiment, the global packet drop rate is used to indicate the global load of the cluster, the CPU utilization and memory utilization is used to indicate the resource utilization of each node. The output of GBESS is the scheduling weights which is further dispatched to the flow scheduler.

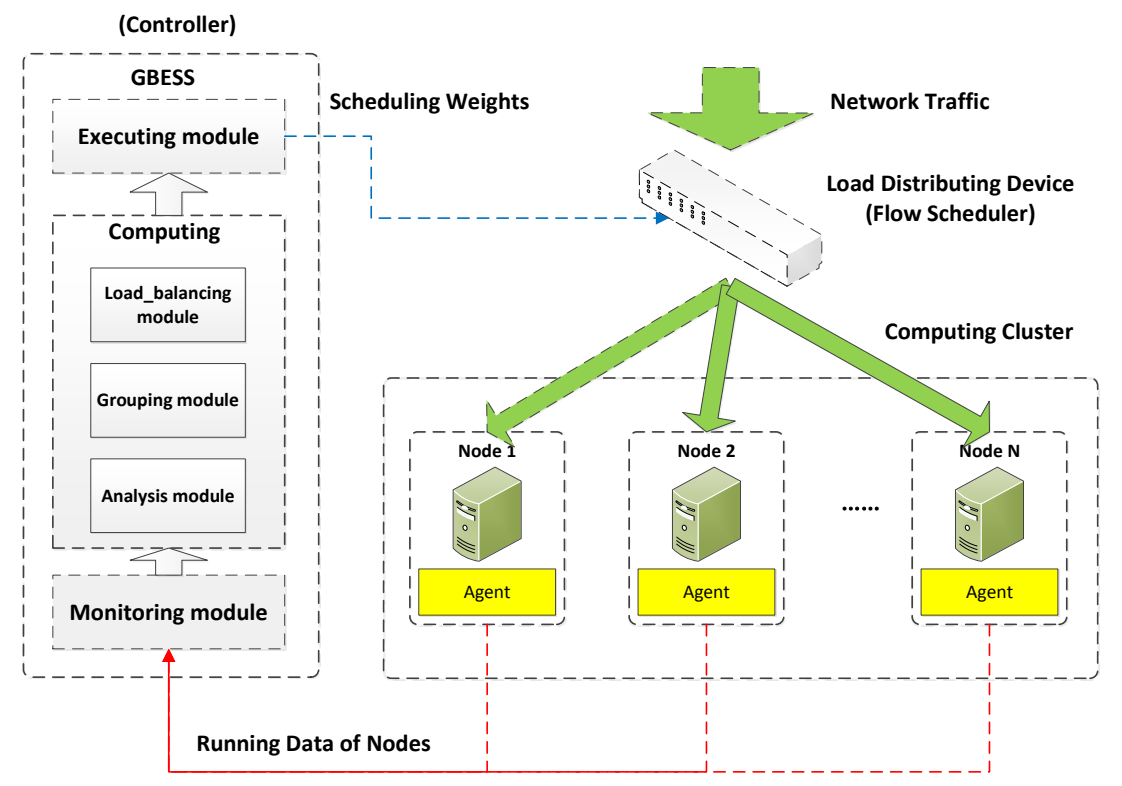

Fig. 3: Experimental testbed.

In this experiment, it sets up two cluster named dynamic group and static group. The dynamic group is scheduled by GBESS and the static group is a comparison group. The node number of each cluster is $N=96$, the input network traffic is $15-20 \mathrm{Gbps}$, and the collecting interval of GBESS is 1 second. Since GBESS tries to save energy by exploiting slack time to shutdown the cold nodes, the number of running cores is used to indicate the energy consumption of the cluster in this experiment. Moreover, the global packet drop rate is also used to indicate the global QoS of the cluster which evaluates the side effect of GBESS.

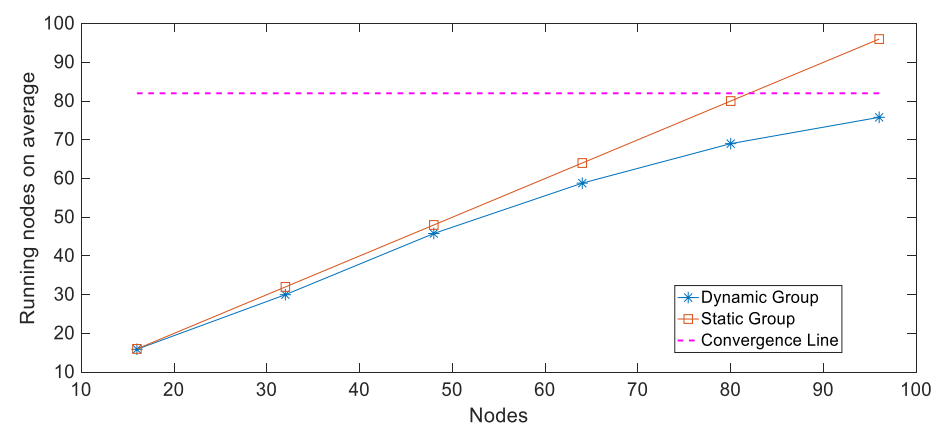

Fig. 4: Energy consumption.

\subsection{Experimental Results}

The experimental results are shown in Fig.4 and Fig.5. Fig.4 illustrates the total energy consumption when the packet drop rate of dynamic group is no higher than static group, namely the QoS of dynamic group is no less than static group. The experimental result presents that the energy saving of dynamic group is $12.1 \%$ compared with static group under the premise of QoS assurance. The energy efficiency of GBESS is improving along with node number increasing. It is $17.7 \%$ better than static group when node number exceeds 64. To be mentioned, the dotted line in Fig. 4 indicates the convergence value of energy saving by GBESS while the cluster scale growing. The convergence value further means the performance lower bound of GBESS under the current network environment. 
Fig.5 illustrates that the average QoS of dynamic group is 7.2\% better than static group, which further indicates the schedulability and QoS guarantee of GBESS.

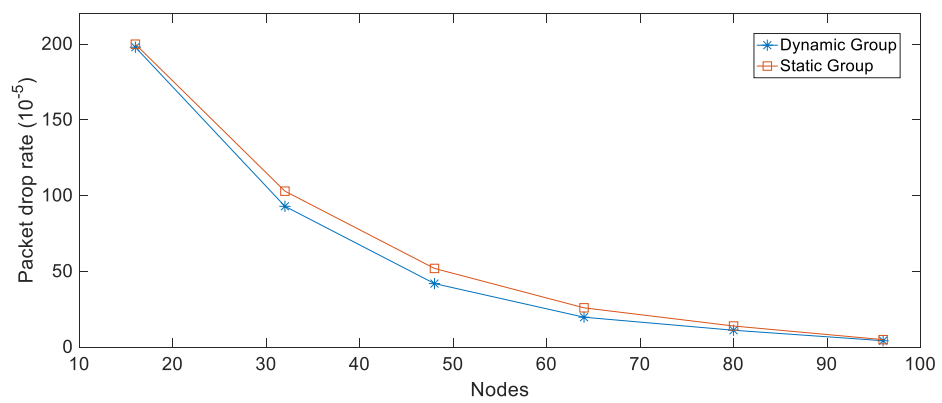

Fig. 5: QoS.

\section{Summary}

This paper presents a Grouping and Balancing based Energy-Saving Scheduling (GBESS). GBESS is a double level strategy: 1) It reduces the energy consumption by grouping the nodes of cluster into hot group and cold group; 2) It creates slack space for energy-saving grouping and ensures the lower bound of QoS by load balancing the hot group. Experimental evaluations show that GBESS can reduce the energy consumption by $12.1 \%$ on average under QoS assurance. Besides, the energy efficiency of GBESS is improving along with the cluster scale growing. The QoS of GBESS is no less than the contrast group, which further indicates the schedulability and QoS guarantee of GBESS.

\section{Acknowledgements}

This work was financially supported in part by the National Natural Science Foundation of China (61402125).

\section{References}

[1] Valentini G L, Lassonde W, Khan S U, et al. An overview of energy efficiency techniques in cluster computing systems[J]. Cluster Computing, 2013, 16(1):3-15.

[2] Abts D, Marty M R, Wells P M, et al. Energy proportional datacenter networks[J]. Acm Sigarch Computer Architecture News, 2010, 38(3):338-347.

[3] J Hamilton. Cost of power in large-scale data centers[EB/OL]. http://perspectives.mvdirona.com.

[4] Liao D, Li K, Sun G, et al. Energy and performance management in large data centers: A queuing theory perspective[C]// International Conference on Computing, Networking and Communications. IEEE, 2015:287-291.

[5] Luo R C, Chen O. Mobile sensor node deployment and asynchronous power management for wireless sensor networks[J]. IEEE Transactions on Industrial Electronics, 2012, 59(5): 2377-2385.

[6] Jiang Q, Xi H S, Yin B Q. Stochastic switching model and policy optimization online for dynamic power management[J]. Acta Automatica Sinica, 2007, 33(1): 66-71.

[7] Salehi M E, Samadi M, Najibi M, et al. Dynamic voltage and frequency scheduling for embedded processors considering power/performance tradeoffs[J]. IEEE Transactions on Very Large Scale Integration (VLSI) Systems, 2011, 19(10): 1931-1935.

[8] K. J. Astrom, and T. Hagglund, 1995. PID Controllers: Theory, Design, and Tuning (2nd). International Society for Measurement and Con.

[9] E. Pinheiro, R. Bianchini, E. V. Carrera, et al, 2003. Dynamic cluster reconfiguration for power and performance. Compliers and Operating Systems for Low Power, 75-93.

[10] Wegener J, Mueller F. A comparison of static analysis and evolutionary testing for the verification of timing constraints [J]. Real-Time Systems, 2001, 21(3): 241-268.

[11] Genova, Z., \& Christensen, K. J. (2000). Challenges in Switching for Implementing Globally Distributed Web Sites, 89-94. 